\title{
EL MENTOR MODELO Y SU INFLUENCIA EN EL DESEMPEÑO LABORAL DE LOS TRABAJADORES DE LA FRANQUICIA DE IDIOMAS UK VIVENTIAL
}

\author{
Keila Flor FUSTAMANTE CASTILLO ${ }^{1}$
}

\begin{tabular}{lll} 
Recibido & $:$ & 10.02 .2020 \\
Aceptado & $:$ & 20.05 .2020 \\
Publicado & $:$ & 06.07 .2020 \\
\hline
\end{tabular}

RESUMEN: El presente trabajo busca determinar si tanto la confrontación y el desafío tienen influencia en el desempeño laboral de los trabajadores de una franquicia de idiomas en San Martín de Porres. Asimismo, para los fines de la investigación esta es de tipo aplicada a nivel descriptivo con un diseño correlacional dado que estable relación entre dos variables: la confrontación y desafío, y, el desempeño laboral, la población y muestra se ha tomado del instituto UK Vivential, la cual se desarrollará a mayor detalle en el presente artículo. Asimismo, para la medición de los resultados se utilizaron como instrumentos cuestionarios enfocados a los trabajadores de la mencionada institución. En principio para la medición se ha utilizado el coeficiente de relación de Spearman, arrojando un resultado aceptable y que la relación entre ambas variables es alta.

Palabras claves: confrontación, desafío, desempeño laboral, influencia

\section{The model mentor and its influence on the job performance of $U K$ Vivential language franchise workers}

\begin{abstract}
The present work seeks to determine if both the confrontation and the challenge influence the labor performance of the workers of a language franchise in San Martín de Porres. Also, for the research, this is of the type applied at the descriptive level with a correlational design since it a stable relationship between two variables: confrontation and challenge, and, labor performance, population and sample has been taken from the UK Vivential institute, which will be further elaborated in this article. Questionnaires were also used to measure the results for the employees of the Institute. In principle the Spearman ratio has been used for the measurement, producing an acceptable result and the relationship between the two variables is high.
\end{abstract}

Keywords: confrontation, challenge, job performance, influence.

\footnotetext{
${ }^{1}$ Universidad Nacional Mayor de San Marcos. Email: keilafustamante@gmail.com
} 


\section{Journal of the Academy $|150|$}

\section{INTRODUCCIÓN}

Actualmente, por los aspectos fundamentales que engloba la modernización y la globalización, el espacio laboral ha sufrido diversos cambios no solo a nivel de su estructura sino también a sus procesos, lo que implica también tener al trabajador como un eje fundamental para el desarrollo de las actividades que deben realizar. Sin embargo, ante esta ola de constantes cambios en el interior de las instituciones, el ambiente laboral sufre también la aparición de fenómenos sociales entre los trabajadores que influyen en su desempeño laboral, en este caso la confrontación de estos problemas y el desafío que esto supone son alternativas que busquen mejorar su desempeño. Es por ello que la aparición del mentor para buscar atender, analizar, evaluar todos los factores que permitan mejorar el rendimiento de los trabajadores es fundamental para el desarrollo de las empresas.

Se considera a un mentor como un sujeto comúnmente mayor y establecido en una organización, que aconseja a los empleados más jóvenes, que funge como entrenador (coach), brinda apoyo social y sirve como tutor.

En sentido amplio, Velaz (2009) señalaba que por mentoría se entiende la relación establecida entre una persona con mayor experiencia en un ámbito (mentor) y otra con menor o ninguna experiencia (mentorizado) con el objetivo de facilitar y desarrollar sus competencias y socialización, incrementando así sus posibilidades de éxito en la actividad a desempeña.

Sin embargo, en la actualidad existe una confusión entre lo que sería el coaching con la mentoría, por lo que es necesario establecer una diferencia.

Figura 1: Diferencia entre coach y mentor

\begin{tabular}{|l|l|l|}
\hline & \multicolumn{1}{|c|}{ Coach } & \multicolumn{1}{|c|}{ Mentor } \\
\hline Objetivo clave & $\begin{array}{l}\text { Corregir comportamientos } \\
\text { inadecuados, mejorar el rendimiento y } \\
\text { entrenar nuevas competencias que el } \\
\text { empleado necesita para asumir nuevas } \\
\text { responsabilidades }\end{array}$ & $\begin{array}{l}\text { Dar apoyo y guía personal al } \\
\text { crecimiento del pupilo }\end{array}$ \\
\hline Iniciativa & el coach dirige el aprendizaje & $\begin{array}{l}\text { Es el pupilo el que está a cargo de } \\
\text { su aprendizaje }\end{array}$ \\
\hline Voluntariedad & $\begin{array}{l}\text { A pesar de que el acuerdo del } \\
\text { subordinado a aceptar el coaching es } \\
\text { esencial, no es necesariamente } \\
\text { voluntario. }\end{array}$ & $\begin{array}{l}\text { Tanto el mentor como el pupilo } \\
\text { participan voluntariamente. }\end{array}$ \\
\hline Foco & $\begin{array}{l}\text { Problemas inmediatos y aprendizaje de } \\
\text { las oportunidades que surjan }\end{array}$ & $\begin{array}{l}\text { Desarrollo de la carrera } \\
\text { profesional a largo plazo }\end{array}$ \\
\hline Roles & $\begin{array}{l}\text { El peso está en lo que dice el coach } \\
\text { con un adecuado feedback }\end{array}$ & $\begin{array}{l}\text { El peso está en el escuchar, } \\
\text { proveer modelos, hacer } \\
\text { sugerencias y facilitar conexiones }\end{array}$ \\
\hline Duración & $\begin{array}{l}\text { Se concentra en necesidades de corto } \\
\text { plazo }\end{array}$ & Es a largo plazo \\
\hline Relaciones & El coach puede ser el jefe del coachee & $\begin{array}{l}\text { La mayoría de expertos } \\
\text { recomiendan que el mentor no esté } \\
\text { en la cadena de mando del pupilo }\end{array}$ \\
\hline
\end{tabular}

Fuente: Elaboración propia

Es entonces de acuerdo a esta diferencia que el mentor es aquel que transfiere su experiencia y conocimientos a otra persona, normalmente más joven o con menor experiencia en determinado ámbito. Es un modelo, consejero, fuente de inspiración y goza de la admiración u respeto del pupilo.

Asimismo, de acuerdo a Cohen (1998) el mentor debe cumplir con diversas competencias, sin embargo, para los fines de la investigación y de la variable, debemos señalar el aspecto el Mentor modelo: 


\section{Journal of the Academy $|151|$}

Figura $\mathrm{N}^{\circ} 2$

Competencias del mentor modelo
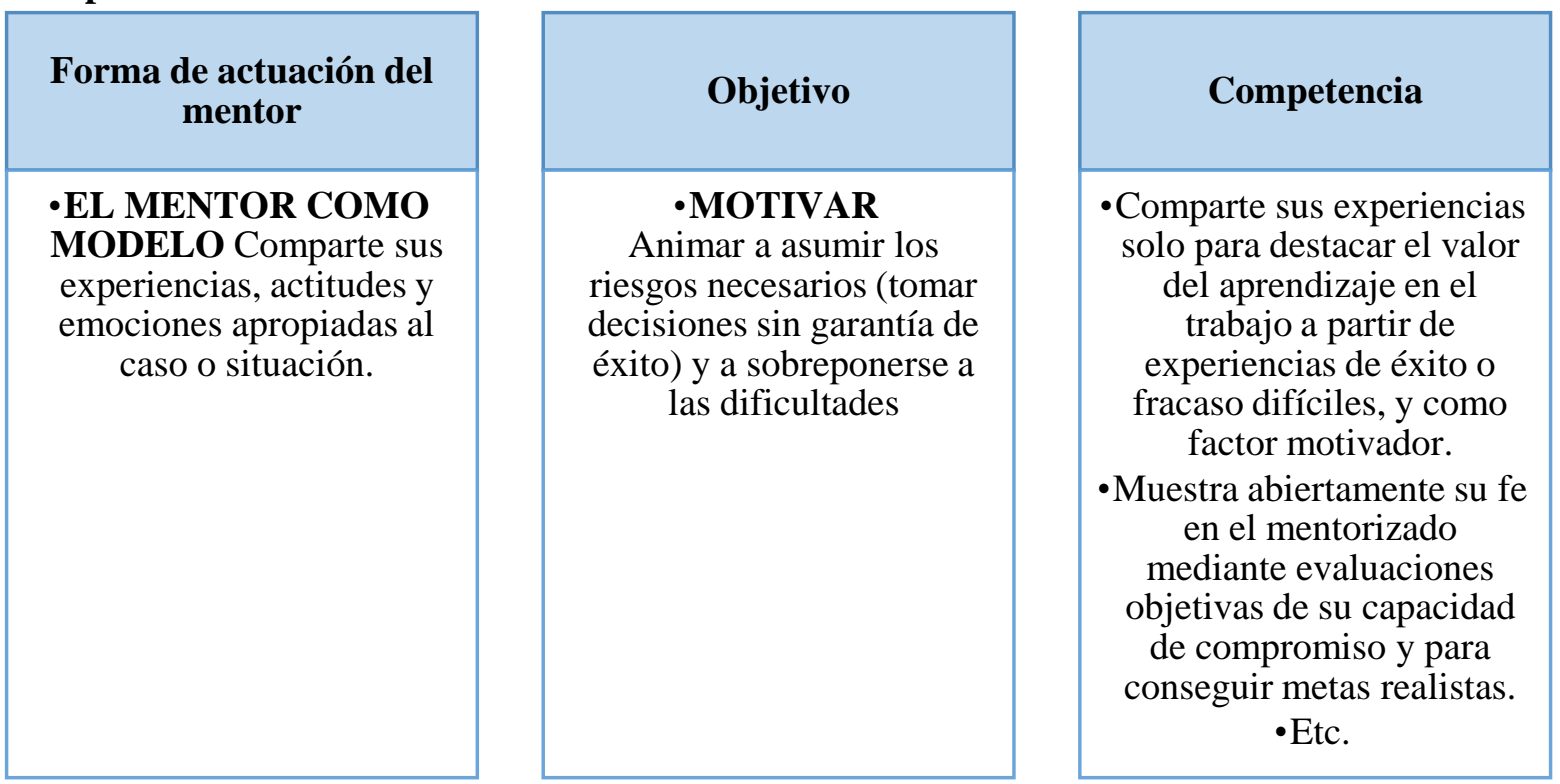

Fuente: Elaboración propia

Asimismo, sobre este proceso, Escobar (2015) señala que:

Esta modalidad supone un desarrollo individualizado y global a largo plazo, que apoya al participante, proporcionándole un bagaje útil en toda su carrera profesional dentro y fuera de la organización. Para lograr los objetivos que se plantean en esta modalidad, se requiere de precisión para encontrar el mentor adecuado para cada participante; de un compromiso por parte del individuo y de la organización; y por último del establecimiento de una buena relación entre el mentor y los participantes.

Es por ello que a través de los programas de Mentoring se busca que existe una guía a un participante de una organización a desarrollarse en un largo plazo que le permita mejorar su desempeño laboral e incluso embelleciendo objetivos a futuros, ya sea en el ámbito que fuera.

Por otro lado, en lo que respecta a la motivación como factor que afecte el desempeño laboral, Espada (2007) señalaba que es un factor emocional básico para el ser humano y para cualquier profesional, estar motivado significa realizar las tareas cotidianas sin apatía y sin un sobreesfuerzo adicional; aunque para unos el trabajo o las actividades de todo orden pueden representar una carga, para otros es un acicate, un revulsivo y a veces una liberación

Asimismo, Ramírez, Abreu y Badii (2008) señalan que diversos autores se han concentrado en la elaboración de diversas clasificaciones que puedan explicar la motivación laboral:

- Campbell, Dunnette y otros establecen teorías más clásicas, la cual establecen criterios fundamentales para la motivación laboral, asimismo, consideran son las necesidades las que determinan la conducta laboral del trabajador.

- Locke se basa en la distinción de teorías basadas en las necesidades, en los valores, teorías de las metas y de la autoeficacia.

- La más actual de Kanfer que propone un modelo heurístico de constructor y teorías motivacionales y agrupa las distintas teorías en función de los constructores que cada una considera centrales para la motivación (por ejemplo: necesidades o intereses, motivos, elección cognitiva, intenciones, objetivos, auto-regulación), ordenando estos constructores desde los más dístales o alejados de la acción concreta a los más proximales o cercanos a esa acción. 


\section{Journal of the Academy $|152|$}

Acerca del desempeño laboral, es importante señala lo mencionado por Chiavenato, I. (2007, p. 256) el cual mención menciona que existen diversas competencias para que los trabajadores en base al desempeño mostrado puedan sostenerse en las organizaciones:

1. Habilidades interpersonales: Se entiende como aquellas necesarias para trabajar en equipo, construir o liderar equipos y facilitar la integración de las personas.

2. Habilidades para resolver problemas: Analizar y utilizar planteamientos para resolver problemas.

3. Habilidades para comunicar: Saber expresarse verbalmente de forma articulada, hacer presentaciones y redactar con corrección.

4. Habilidades para planear y organizar: Ser hábil para administrar el tiempo y alcanzar objetivos.

5. Responsabilidad: Tomar la iniciativa, aceptar responsabilidades y tareas adicionales para el bien del grupo.

6. Asertividad: Ser capaz de comunicar de forma abierta y directa, demostrar confianza en uno mismo y prestar atención a la percepción de otros.

7. Flexibilidad: Ser capaz de adaptarse a los cambios que sufre la organización, de aceptar nuevas ideas, de implantar nuevas maneras de hacer las cosas.

8. Juicio: Ser capaz de determinar el nivel adecuado de riesgo y la acción correcta, tomar decisiones significativas.

En ese sentido, se procederá de tallar la investigación realizada si como arribar a la confirmación de la hipótesis planteada al inicio del presente trabajo y materia de investigación.

\section{DESARROLLO}

\section{Material y métodos}

\section{Diseño y lugar}

Se ha realizado una investigación de tipo No experimental - Descriptivo, ya que no se construyó ninguna situación, ni hubo manipulación de la misma, para estudiar efectos, sino que se observó una situación ya existente.

Asimismo, señalar que esta investigación es correlacional debido a que la información fue recogida a partir de la observación directa de las características que se presenta las variables con la finalidad de determinar y validar su de relación. Es importante destacar que el se ha dirigido a la institución de idiomas UK Vivential la cual será analizada para validar la relación de las variables materia de investigación.

\section{Población}

Se ha enfocado principalmente a una totalidad de 62 trabajadores entre directivos, docentes y personal administrativo de la franquicia de idiomas UK Vivential. Es necesario precisar que la institución ha brindado su autorización para el estudio realizado.

\section{Instrumento de recolección de datos}

La fuente de información en el estudio fue primaria y personal, se tuvo acceso inmediato para la recolección de datos.

Para la recolección de datos se utilizó una encuesta estructurada que contiene ítems que aborda las variables, la cual cuenta que contiene la batería de preguntas en base a los indicadores de la variable. Para la variable independiente y dependiente, la encuesta estructurada estuvo dirigido a los docentes, directivos y personal administrativo; 


\section{Journal of the Academy $|153|$}

Estos instrumentos han sido validados por expertos, 03 expertos en este caso, En el estudio, para la validez y la confiabilidad del instrumento se empleó; una prueba piloto y la opinión de expertos; a través de una ficha.

\section{Variables}

Variable 1: Mentor modelo

El mentor modelo básicamente se trata de un lazo más fuerte, porque se encuentra casi al final del camino de este proceso, se motiva, se ayuda a los aprendices gracias a las experiencias de los mentores.

Lisfshitz (2017) señala que:

El término "mentor" suele usarse como sinónimo de maestro o profesor; si bien algunos lingüistas consideran que en realidad no existen los sinónimos, sino que cada término tiene sutilezas que lo diferencian de los otros que se utilizan de manera intercambiable. (p. 147)

Dimensión:

- Motivación

- Auto-revelación

- Divulgación

- Lidiar con el Riesgo

- Personalice la Relación

Variable 2: Desempeño laboral

Chiavenato (2002, p.236) considera que "el Desempeño es la eficacia del personal que trabaja dentro de las organizaciones, la cual es necesaria para la organización, funcionando el individuo con una gran labor y satisfacción laboral"

Dimensión:

- Desarrollo organizacional

- Comportamiento organizacional

\section{Resultados}

Tabla $\mathbf{N}^{\circ} 1$

Resultado de Directivos

\begin{tabular}{|l|l|l|l|l|l|}
\hline \multicolumn{2}{|c|}{} & Frecuencia & Porcentaje & Porcentaje válido & $\begin{array}{l}\text { Porcentaje } \\
\text { acumulado }\end{array}$ \\
\hline \multirow{4}{*}{ Válido } & Malo & 1 & 25,0 & 25,0 & 25,0 \\
\cline { 2 - 6 } & Regular & 1 & 25,0 & 25,0 & 50,0 \\
\cline { 2 - 6 } & Bueno & 2 & 50,0 & 50,0 & 100,0 \\
\cline { 2 - 6 } & Total & 4 & 100,0 & 100,0 & \\
\hline
\end{tabular}

Fuente: Datos procesados mediante el software SPSS 24 y Excel.

Elaborado: Por la responsable de la investigación. 


\section{Journal of the Academy $|154|$}

\section{Figura $\mathbf{N}^{\circ} 3$}

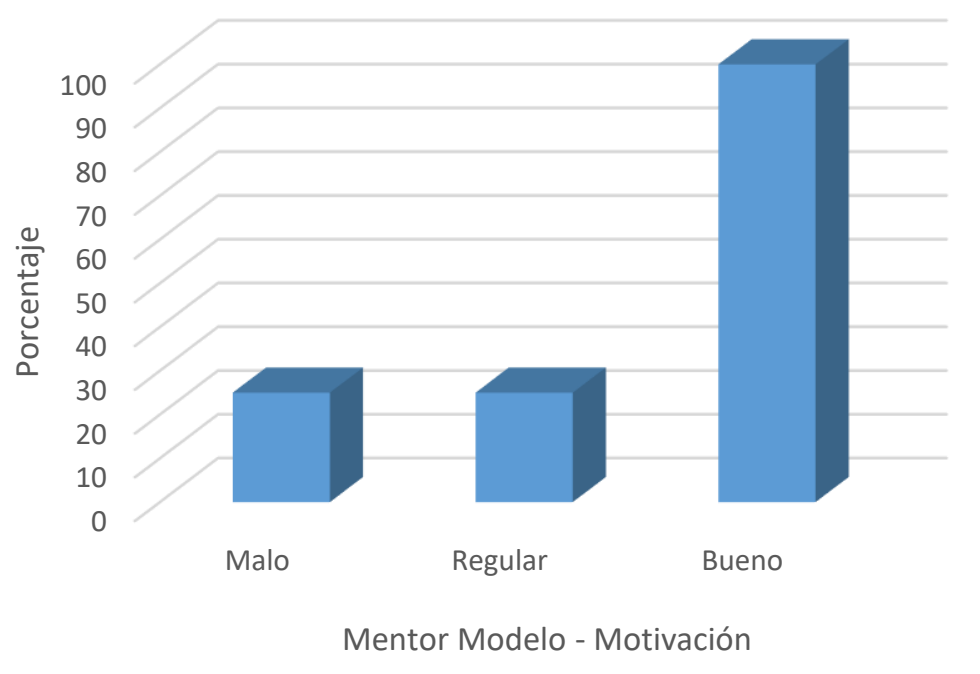

Interpretación: En la tabla y gráfico estadístico; se evidencia los 4 Directivos encuestados, representada por el 50\% manifiestan que es bueno el mentor Modelo - Motivación, el 25\% señala que es regular y el $25 \%$ manifiesta que es malo.

Tabla $\mathbf{N}^{\circ} 2$

Resultados de los Docentes

\begin{tabular}{|l|l|l|l|l|l|}
\hline \multicolumn{2}{|c|}{} & Frecuencia & Porcentaje & Porcentaje válido & $\begin{array}{l}\text { Porcentaje } \\
\text { acumulado }\end{array}$ \\
\hline \multirow{4}{*}{ Válido } & Malo & 1 & 5,6 & 5,6 & 5,6 \\
\cline { 2 - 6 } & Regular & 6 & 33,3 & 33,3 & 38,9 \\
\cline { 2 - 6 } & Bueno & 11 & 61,1 & 61,1 & 100,0 \\
\cline { 2 - 6 } & Total & 18 & 100,0 & 100,0 & \\
\hline
\end{tabular}

Fuente: Datos procesados mediante el software SPSS 24 y Excel.

Elaborado: Por la responsable de la investigación.

\section{Figura $\mathbf{N}^{\circ} 4$}

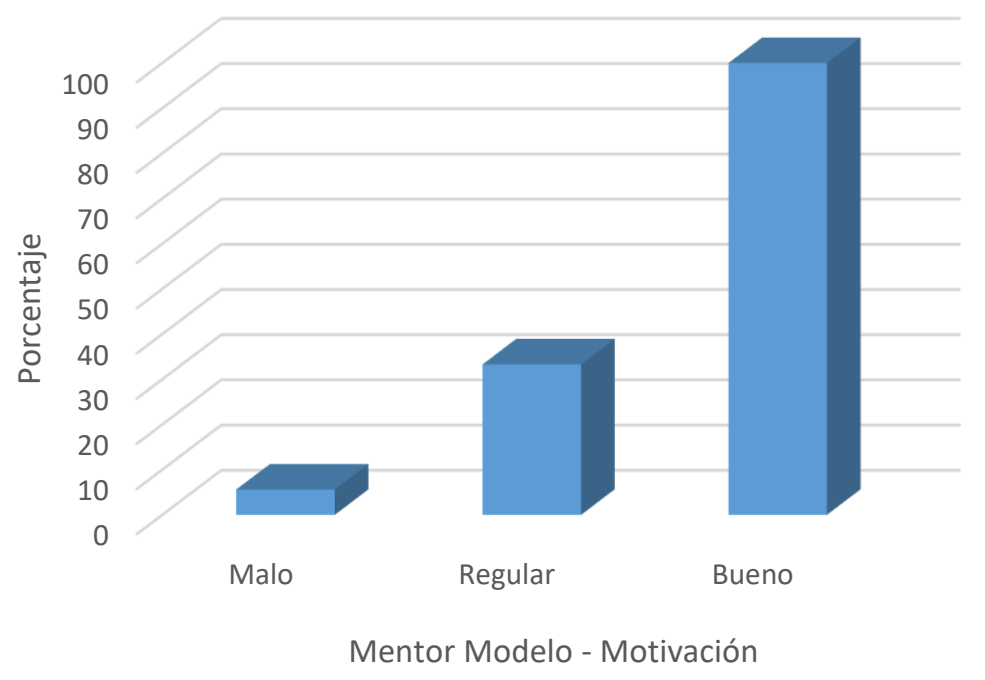


Interpretación: En la tabla y gráfico estadístico; se evidencia los 18 docentes encuestados, representada por el 61,1\% manifiestan que es bueno el Mentor Modelo - Motivación, el 33,3\% señala que es regular y el 5,6\% manifiesta que es malo.

\section{Tabla $\mathbf{N}^{\circ} 3$}

\section{Resultados del Personal Administrativo}

\begin{tabular}{|l|l|l|l|l|l|}
\hline \multicolumn{2}{|c|}{} & Frecuencia & Porcentaje & $\begin{array}{l}\text { Porcentaje } \\
\text { válido }\end{array}$ & $\begin{array}{l}\text { Porcentaje } \\
\text { acumulado }\end{array}$ \\
\hline \multirow{4}{*}{ Válido } & Malo & 5 & 26,3 & 26,3 & 26,3 \\
\cline { 2 - 6 } & Regular & 7 & 36,8 & 36,8 & 63,2 \\
\cline { 2 - 6 } & Bueno & 7 & 36,8 & 36,8 & 100,0 \\
\cline { 2 - 6 } & Total & 19 & 100,0 & 100,0 & \\
\hline
\end{tabular}

Fuente: Datos procesados mediante el software SPSS 24 y Excel.

Elaborado: Por la responsable de la investigación.

\section{Figura $\mathbf{N}^{\circ} 5$}

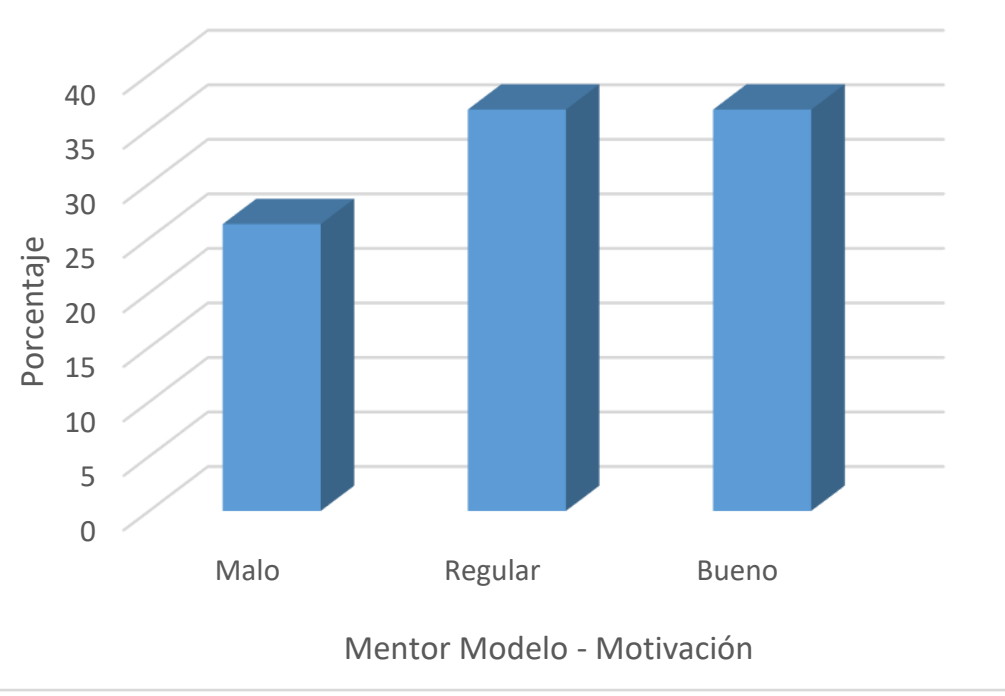

Interpretación: En la tabla y gráfico estadístico; se evidencia los 19 miembros del Personal Administrativo encuestados, representada por el 36,8\% manifiestan que es bueno el Mentor Modelo Motivación, el 36,8\% señala que es regular y el 26,3\% manifiesta que es malo.

\section{Discusión de resultados}

La presente investigación permitió responder al objetivo general: Determinar la influencia que existe del mentor en el desempeño laboral de los trabajadores de la franquicia de idiomas UK Vivential. En el análisis del presente estudio, se determinó estadísticamente un coeficiente de correlación muy buena de $\mathrm{r} \mathrm{P}=.894$, entre las variables el mentor y desempeño laboral en la población estudiada. Se determinó respuestas divididas de parte de la muestra, representadas en la variable el mentor cuyas respuestas resolvieron que en un mayor porcentaje se considera como buena.

Asimismo, para la variable desempeño laboral las respuestas también se encuentran distanciadas, el $50 \%$ opinan que es regular, mientras que un $25 \%$ malo y $25 \%$ es bueno. Estos resultados se analizan con los de los investigadores Núñez-Cacho \& Grande (2012) en sus resultados muestran la existencia de un efecto causal y directo de la aplicación del mentor y el rendimiento de las empresas. De este modo se comprueba que los planteamientos teóricos formulados y contribuimos a un conocimiento más 


\section{Journal of the Academy $|156|$}

profundo de esta Intangible Capital práctica, sus condiciones de efectividad y los beneficios que reporta a la empresa.

De acuerdo a los resultados obtenidos Sum (2015) durante la investigación determinan que la motivación influye en el desempeño laboral de los colaboradores. Quien señala que la motivación es de gran relevancia si se desea tener un buen desempeño de los colaboradores, ya que es la misma la que lleva a que el individuo actué de cierta manera, en una organización se pretende alcanzar los objetivos establecidos y el cumplimiento de las metas y así traerá grandes beneficios. El desempeño de los colaboradores se ve reflejado en las actividades que ellos realizan en su área de trabajo, todo esto depende también de la forma y la manera que los colaboradores de una organización estén motivados.

Respecto a esta hipótesis, las estadísticas determinaron un coeficiente de correlación buena de $\mathrm{r} \mathrm{P}=$ .781, entre la mentor modelo, motivación y la variable desempeño laboral. Encontrándose diferencias en las respuestas de la muestra, en cuanto a la dimensión mentor modelo, motivación las respuestas determinaron que el $61.1 \%$ lo califica de regular frente a un $33.3 \%$ como malo y el 5,6\% señala como Bueno.

De igual forma, para la variable desempeño laboral las respuestas se encuentran distanciadas, el 50\% opinan que es bueno, el $25 \%$ señalan que es regular mientras que $25 \%$ señala que es malo. Entre tanto, Cárdenas \& Villacorta (2017) determinan la relación entre mentoring y desempeño laboral, encontrando una relación directa entre ellos, pues el resultado es 0.712 , lo que confirma una correlación directa, que tiene una similitud a los resultados de la presente investigación realizada.

\section{CONCLUSIONES}

Tras analizar la influencia que existe del Mentor Modelo-Motivación en el desempeño laboral de los trabajadores de la franquicia de idiomas UK Vivential, El resultado se evidencia estadísticamente la correlación es de 0.904, donde demuestra que existe una relación positiva alta; es decir que a medida que se incrementa la relación en un mismo sentido, crece para ambas variables. Asimismo, la significatividad, es alta porque la evidencia estadística demuestra que los resultados presentan un menor a 0.01. Entonces no existe suficiente evidencia estadística para rechazar la relación, porque la p-valor $<0.05$.

En ese sentido, es necesario que el mentor debe concientizar a sus protegidos de que el aprendizaje es una responsabilidad personal y que todos necesitan aceptar de dicha responsabilidad para su aprendizaje y desempeño.

Finalmente, es necesario que se forme a los Mentores en técnicas, actitudes y habilidades de Mentoring antes de iniciar un programa de este tipo en la institución.

\section{REFERENCIAS BIBLIOGRÁFICAS}

Cárdenas, M., Villacorta, E. (2017) Tesis: "Mentoring y desempeño laboral en la piladora de arroz Rey León - Morales año 2015” Universidad nacional de San Martin- Tarapoto, Perú.

Cohen, N.H. (1998): Perfil de competencias del mentor: manuela del monitor, autodiagnóstico y cuaderno de evaluación. Madrid, Editorial Universitaria Ramón Areces.

Chiavenato, I. (2007). Administración de recursos humanos: el capital humano de las organizaciones. México: McGraw HILL.

Escobar, M. (2005). LAS COMPETENCIAS LABORALES: ¿La estrategia laboral para la competitividad de las organizaciones?. Estudios Gerenciales, 21(96), 31-55. Retrieved June 02, 2020, 


\section{Journal of the Academy | $157 \mid$}

from

http://www.scielo.org.co/scielo.php?script=sci_arttext\&pid=S0123$59232005000300002 \& \operatorname{lng}=$ en\&tlng=es.

Espada, M. (2006). Nuestro motor emocional: la motivación. Editorial Díaz de Santos.

Lifshitz, A. (2017). Mentores. Medicina interna de México, 33(2), 147-149. Recuperado en 02 de junio de 2020, de http://www.scielo.org.mx/scielo.php?script=sci_arttext\&pid=S0186$48662017000200147 \& \operatorname{lng}=\mathrm{e} \&$ tlng=es.

Núñez-Cacho, P. \& Grande, F. (2012). El desarrollo de los recursos humanos a través del mentoring: El caso español. Universidad de Jaén (Spain).

Ramírez, R. D., Abreu, J. L., \& Badii, M. H. (2008). La motivación laboral, factor fundamental para el logro de objetivos organizacionales: Caso empresa manufacturera de tubería de acero. Revista Daena (International Journal of Good Conscience), 3(1).

Vélaz, C. (2009). COMPETENCIAS DEL PROFESOR-MENTOR PARA EL ACOMPAÑAMIENTO AL PROFESORADO PRINCIPIANTE. Profesorado. Revista de Currículum y Formación de Profesorado, 13(1),209-229.[fecha de Consulta 2 de Junio de 2020]. ISSN: 1138-414X. Disponible en: https://www.redalyc.org/articulo.oa?id=567/56711733015 